\title{
The Skin Whitening Effect of Co-Cultured Conditioned Medium: Involvement of Synergy between Stem Cells and Immune Cells
}

\author{
Tae-Kyung Ko', Hye-Mi Hong1, Dong-Soo Kim², Da-Young Yoo', Sung Hwan Hwang1, \\ Jung-Hwa Kang²
}

${ }^{1}$ Research \& Development, Smart Cell Lab. Co., Ltd., B2, International St. Mary’s Hospital, Incheon, Korea

${ }^{2}$ Research \& Development, Immunisbio Co., Ltd., B2, International St. Mary’s Hospital, Incheon, Korea

Email: ktk5817@smartcelllab.com, kjunghwa@immunisbio.com

How to cite this paper: Ko, T.-K., Hong, H.-M., Kim, D.-S., Yoo, D.-Y. Hwang, S.H. and Kang, J.-H. (2021) The Skin Whitening Effect of Co-Culture Medium: Involvement of Synergy between Stem Cells and Immune Cells. Journal of Cosmetics, Dermatological Sciences and Applications, 11, 342-355.

https://doi.org/10.4236/jcdsa.2021.114028

Received: October 26, 2021

Accepted: December 11, 2021

Published: December 14, 2021

Copyright $\odot 2021$ by author(s) and Scientific Research Publishing Inc. This work is licensed under the Creative Commons Attribution-NonCommercial International License (CC BY-NC 4.0). http://creativecommons.org/licenses/by-nc/4.0/ (c) (i) (8) Open Access

\begin{abstract}
Many researchers have described that mesenchymal stem cells conditioned medium and immune cells conditioned medium have a clear whitening effect when they are used as cosmetic ingredients. In this study, we confirmed the whitening efficacy of various concentrations of immune cells and stem cell conditioned media. The author tried to study a conditioned medium that has a strong whitening effect even with a composition of less than $20 \%$ (the most used concentration in cosmetics). Because of the fact that the conditioned medium contains various cytokines and growth factors secreted by stem cells or immune cells, it is known to have effects such as wound healing, antioxidant, and whitening effect. Recently, stem cells have been used not only in the development of cosmetic raw materials but also in skincare procedures, and there are reports being released of cosmetics using immune cells conditioned medium. The concentration-dependent whitening effect equivalently increased as the concentration of the mono-cultured conditioned medium was obtained through the stem cells or immune cells culture. In the case of co-culture, whitening results are like the effect of positive control such as arbutin in the medium carrying only $10 \%$ of the co-cultured conditioned medium. It is possible that enhanced whitening efficiency in co-cultured conditioned medium leads to a major innovation in the global cosmetic market.
\end{abstract}

\section{Keywords}

Stem Cell, Immune Cell, Natural Killer Cell (NK Cell), Conditioned Medium, Co-Cultured, Skin Whitening 


\section{Introduction}

The history of skin whitening is very long, and even today, many people crave bright skin. Whitening of the skin shows its position in society or simply serves as a standard of beauty. Despite some negative perceptions related to skin whitening, even today, the craving for skin whitening is one of the cosmetic trends that men, as well as women, want. Despite the harm to the skin caused by excessive obsession and desire for whitening, and various regulations, interest in skin whitening is not diminishing [1].

The color of the skin is mainly determined by the content of a pigment called melanin in the skin, and this melanin is biosynthesized by melanocytes in the basal layer of the skin, and through the cytoplasmic process, it moves from the basal layer of the epidermis to the stratum corneum by the keratinization process [2] [3].

In the recent cosmetics market, immune cells conditioned medium, or stem cells conditioned medium is widely used as a cosmetic factor for whitening human skin due to the melanin synthesis mechanism caused by various factors such as UV exposure [4].

Looking at the process of melanin formation, Tyrosinase is a major mediator that converts tyrosine to DOPA or to DOPA-quinone. Therefore, by inhibiting the activity of this enzyme and inhibiting the production of melanin, the skin whitening effect can be confirmed. In particular, Arbutin, the most well-known whitening agent, is an inhibitor that competes with L-Tyrosine [5] [6], and Kojic acid inhibits the process of tyrosine to DOPA and DOPA to DOPA quinone by acting on the tyrosinase active site.

Most of the researches on skin whitening use plant extracts and various materials are being tried for differentiation in recent years. Recently, along with the interest in cell therapy products, many cosmetics are being developed for the efficacy of cell culture solutions such as stem cells conditioned media.

Mesenchymal stem cells (MSC) are well known for secreting various growth factors such as PDGF, VEGF and EGF, etc. [7]. Multiple studies have demonstrated that mesenchymal stem cells promote hair growth [8] and wound healing [9]. In addition, it is known to have antioxidant [10], anti-wrinkle [11], and whitening [12] [13] effects. Moreover, it has been shown that adipose-derived mesenchymal stem cell conditioned medium protects dermal fibroblasts exposed to oxidative stress, chemical treatment and UV-B [14]. Stem cells are used not only in the development of raw materials for cosmetics but also in the fields of skin beauty treatment and plastic surgery. Recently, cosmetics ingredients through immune cells conditioned medium have been developed as well as commercialized [15].

Natural killer cells (NK cells) are a type of immune cell and are known to be particularly responsible for innate immunity [16]. NK cells are responsible for not only anti-cancer immunity but also defense mechanisms against infections such as viruses and bacteria by receptor-ligand interaction without stimulation of specific antigens [17]. NK cells play a role in killing abnormal cells not only 
through their own receptor-ligand interactions but also by secretomes such as cytokines and chemokines secreted by the cells [18].

There are studies confirming that various cytokines and growth factors are secreted by mutual influence between immune cells and stem cells through co-culture of both cells. Studies have shown that the pattern of secretomes such as IFN-r and TNF-a secreted by NK cells changes when stem cells and immune cells are co-cultured.

However, the development of cosmetics through co-culture of stem cells and immune cells has not yet been developed because of the complexity of the culture process and the difficulty in standardizing the culture [19].

In this study, it was confirmed whether the whitening effect was higher in the co-cultured conditioned medium as well as the stem cells conditioned medium and immune cells conditioned medium. It was confirmed that the secretion of various cytokines and growth factors increased through the synergistic effect of co-culture of stem cells and immune cells. The increased efficacy of whitening was confirmed by measuring cytokines \& growth factors array, melanin contents, and tyrosinase inhibition in the co-cultured conditioned medium rather than the stem cells conditioned medium and immune cells conditioned medium. Through this, it was attempted to confirm the possibility of a cosmetic composition of a co-cultured conditioned medium of stem cells and immune cells having an increased whitening effect.

\section{Materials and Methods}

\subsection{Stem Cells Conditioned Medium Collection}

Stem cells have been purchased at Cefobio (CEFO-ADMSC) and cultured in media containing high-glucose DMEM (Hyclone, SH30243.01), 10\% FBS (Hyclone, SH30109.03) and 1\% AA (Gibco, 15240062) until 80\% confluence. After removing the media, the cells were washed twice with DPBS and the media was replaced with low-glucose DMEM (Sigma, D2902) supplemented with 1\% Serum Replacement 3 (sigma, S2640-100ML) and cultured for 3 days. Thereafter, the conditioned medium was collected and centrifuged at $1800 \mathrm{rpm}$ for $5 \mathrm{mi}$ nutes, followed by 0.22 um filtration.

\subsection{Immune Cells Conditioned Medium Collection}

PBMCs were isolated from blood and washed with normal saline, thereafter the cell suspension was centrifuged at $400 \mathrm{~g}$ for 10 minutes, obtained cells were cultured at $37^{\circ} \mathrm{C}$ and $5 \% \mathrm{CO}_{2}$ for 2 days in KBM502 media containing NK sol (IMMUNISBIO. Co. ltd.) with $2.0 \times 10^{7}$ cells/flask. After this, subculture was performed at intervals of 2 - 3 days. After 14 days of culturing, NK cells were centrifuged at $400 \mathrm{~g}$, and the supernatant was collected to obtain immune cells conditioned medium.

\subsection{Co-Cultured Conditioned Medium Collection}

Stem cells were grown in high-glucose DMEM with $10 \%$ FBS and $1 \%$ AA. When 
the confluency reached about $60 \%$, cells were washed and the ratio of stem cells and immune cells was calculated to be 3:1 and cultured at 0.05\% albumin (Sigma, A4256) in RPMI (Welgene, LM001-01) for 3 days.

The co-cultured conditioned medium was collected, centrifuged for 5 minutes at $1800 \mathrm{rpm}$ and $0.22 \mu \mathrm{m}$ filtration was performed.

\subsection{Cytokine Array}

Stem cells, NK cells, and co-cultured cells were tested according to the manual using RayBio ${ }^{\circledR}$ C-Series Human Cytokine Antibody Array C5 kit. Briefly, each cytokine array membrane was blocked with blocking buffer at room temperature for 30 minutes and reacted overnight at $4^{\circ} \mathrm{C}$ with each conditioned medium and control (only medium). Washed with wash buffer and reacted with biotinylated antibody cocktail. After washing, the reaction was performed overnight at $4^{\circ} \mathrm{C}$ using HRP-streptavidin solution. After the reaction was completed, the membrane was washed and the detection mixture buffer was reacted at room temperature for detection using Davinchi-chemidoc.

\subsection{Growth Factor Array}

Stem cells, NK cells, and co-cultured cells were tested using the Human Growth Factor Antibody Array C1 kit according to the manual. Briefly, the membrane was blocked at room temperature using a blocking buffer. After washing, each conditioned medium and control (only medium) were reacted overnight at $4^{\circ} \mathrm{C}$. After washing, each membrane was treated with biotinylated antibody cocktail and reacted overnight at $4^{\circ} \mathrm{C}$. After washing, each membrane was reacted with HRP-streptavidin, and detection was performed with Davinchi-chemidoc using a detection buffer.

\subsection{B16F10 Cells Culture}

B16F10, a mouse melanoma cell line has been purchased at ATCC (PCS-200-013) and was used as the cells for whitening test, it was prepared to be $5 \times 10^{4} /$ well in a 6 well plate, and Passage 10 was used during seeding. As a conditioned medium, DMEM (Hyclone, SH30243.01) containing 10\% FBS (Hyclone, SH30109.03) and $1 \%$ AA (Gibco, 15240062) was used in $2 \mathrm{~mL}$ each well and incubated at $5 \% \mathrm{CO}_{2}$, $37^{\circ} \mathrm{C}$ for 48 hours in Incubator.

The media was replaced with $2 \mathrm{~mL}$ of DMEM (Hyclone, SH30243.01) containing 0.5\% FBS (Hyclone, SH30109.03) and 1\% AA (Gibco, 15240062) and incubated at $37^{\circ} \mathrm{C}, 5 \% \mathrm{CO}_{2}$ for 24 hours. Thereafter, the co-cultured conditioned medium was treated with $0 \%, 3 \%, 5 \%, 10 \%, 20 \%, 50 \%$, and $1 \mathrm{mg} / \mathrm{mL}$ of Arbutin (Sigma, A4256) was used as a positive control. Total $3 \mathrm{~mL}$ to each well was contained and incubated in Incubator at $37^{\circ} \mathrm{C}$ under $5 \% \mathrm{CO}_{2}$ for 48 hours.

\subsection{Melanin Contents Assay \& Tyrosinase Inhibition Activity Assay}

B16F10 cells treated with mono-cultured or co-cultured conditioned medium 
were washed twice with $4^{\circ} \mathrm{C}$ cold DPBS (Hyclone, LB001-02), and treated with $100 \mu \mathrm{L}$ of $0.1 \mathrm{M}$ Sodium phosphate (Sigma, 71500-250G) buffer ( $\mathrm{pH} 6.8$ ), 1\% triton $\mathrm{x}-100$ (BIO-RAD, 1610407) per well and spread evenly in a well plate. The cells were scraped with a cell scraper (SPL, 90020), collected in an ep-tube (Axygen, MCT-150-C), vortexed for 15 seconds, and stored on ice for 7 minutes. Thereafter, centrifugation was performed for 25 minutes at a speed of 13,000 rpm using a centrifuge set at $4^{\circ} \mathrm{C}$, in advance. Among the separated pallet and supernatant, supernatant was reacted with tyrosinase solution for measuring tyrosinase inhibition, and pallet was used for intercellular melanin contents analysis. After dispensing $130 \mu \mathrm{L}$ of $1 \mathrm{~N} \mathrm{NaOH}$ (Duksan, 1636) containing 10\% dimethyl sulfoxide (Sigma, D2660-100ML) to the harvested pellet, vortexed for 15 seconds, and used a heat block set at $95^{\circ} \mathrm{C}$, in advance for 20 minutes, to dissolve melanin. Samples were diluted 2 times and placed into 96 well plates at $100 \mu \mathrm{L}$ in duplicates. The content of melanin was analyzed by measuring the absorbance at a wavelength of $405 \mathrm{~nm}$ using a Microplate reader.

After measuring the protein content through BCA assay using the harvested supernatant, it was dispensed into a 96 well plate (SPL, 30096) so that the same amount of protein $(30 \mathrm{ug} / \mathrm{mL})$ was in all samples. The rest was filled with DPBS (Hyclone, LB001-02) so that all samples had the same amount. A mixture of 3 $\mathrm{mg} / \mathrm{mL}$ of L-DOPA (3,4-dihydroxy-L-phenylalanine) (Sigma, D9628) in $0.1 \mathrm{M}$ sodium phosphate (Sigma, 71500-250G) of pH 6.8 was dispensed into a 96 well plate (SPL, SPL, 30096) at $180 \mu \mathrm{L}$ each, and heated at $37^{\circ} \mathrm{C}$ for 3 hours. After incubation, color change was observed with the naked eye, and the absorbance was measured at $405 \mathrm{~nm}$ with a Microplate reader. The experiment was independently repeated 3 times.

\subsection{Statistical Analysis}

Significant differences were obtained using the t-test for equidispersive data and using the Welch $\mathrm{t}$-test and SPSS for unequal variance. A p value less than 0.05 was considered statistically significant.

\section{Results}

\subsection{Cytokine \& Growth Factor Array}

Cytokine array was used to find different expression pattern of Cytokines depending on various conditioned medium. Serpin E, one of the Cytokine, was expressed in the conditioned medium obtained through mono-culture of MSC. Otherwise, MIP and IL-2 were expressed in the conditioned medium obtained by mono-culture of immune cells (Figure 1).

It was confirmed that each cytokine was more strongly expressed in the conditioned medium obtained by co-culturing stem cells and immune cells from Cytokine array. In addition, Expression of CCL1, CCL2, MIP-1a, CCL5, CXCL1, CXCL10, IL-8, and IL-6 was detected in the conditioned medium obtained through MSC and immune cells. 


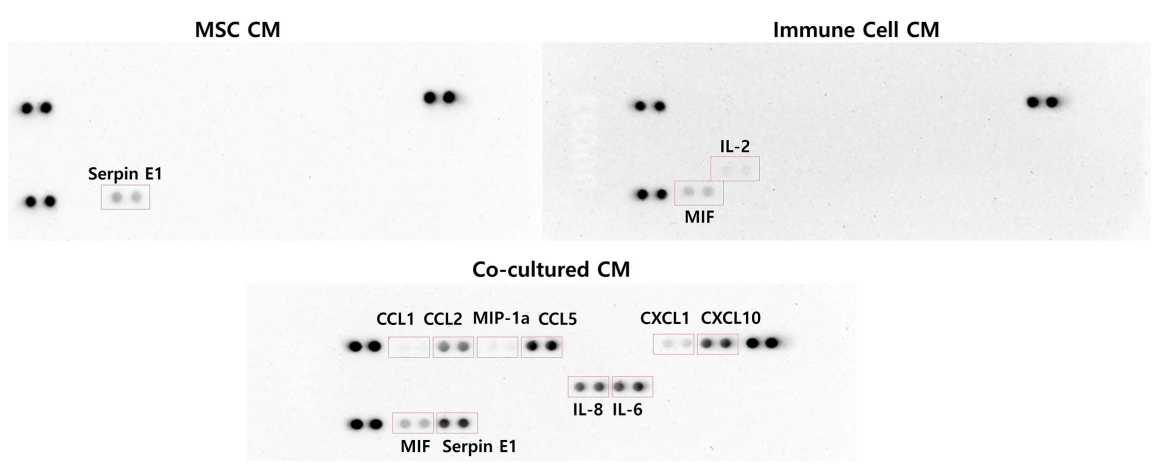

Figure 1. Cytokine array. In the case of the cytokine array, the protocol provided by the company was used. Briefly, each culture was reacted with a membrane, reacted with an antibody, and detected with chemidoc using HRP-streptavidin solution. More types of cytokines were detected in the conditioned medium obtained through co-culture.

To distinguish the different expression patterns of cytokines, growth factor array was performed with three different conditioned medium.

As a result of Growth Factor Assay, most of the growth factors were expressed both in the conditioned medium obtained through mono-culture of MSC or immune cells (Figure 2).

However, some of the growth factors expressed in the immune cells conditioned medium tended to be more expressed in the stem cell conditioned medium, and the expression rate of HGF in the conditioned medium obtained by co-culture of the stem cells and immune cells was approximately $124 \%$ higher than the average of the values in the mono-culture. Moreover, it was confirmed that the expression of IGFPB-4 and IGFPB- 6 was more than twice as high as $221 \%$ and $210 \%$ in the conditioned medium obtained by co-culture of the stem cells and immune cells.

In addition, it was confirmed that the expression rate of M-SCF and MCSF in the conditioned medium obtained by co-culture was respectively $144 \%$ and $134 \%$ higher than the average of each expression rate value in the mono-culture.

\subsection{Melanin Contents Assay \& Tyrosinase Inhibition Activity}

According to the melanin contents assay results, it was found that the melanin synthesis inhibitory effect was maximized as the concentration of the conditioned medium increased in the case of the conditioned medium obtained by mono-culture of stem cells. The amount of melanin synthesis significantly decreased to $91 \% \pm 2 \%$ in the $3 \%, 84 \% \pm 1 \%$ in the $5 \%$, and $75 \% \pm 2 \%$ in the $10 \%$ treated condition. In addition, since it was confirmed to be $67 \% \pm 1 \%$ under the condition of $20 \%$, a continuous decrease was confirmed according to the treatment concentration (Figure 3).

In the case of the conditioned medium obtained by mono-culture of immune cells, it was also verified that the melanin synthesis inhibitory effect tended to decline in $10 \%$ treated group. The amount of melanin synthesis decreased to $92 \% \pm 1 \%$ in the $10 \%$ condition, $86 \% \pm 7 \%$ in the $20 \%$, and $56 \% \pm 6 \%$ in the $50 \%$ treated condition (Figure 4). 
It was confirmed that the melanin synthesis amount significantly decreased depending on the contents of immune cells conditioned medium.

As for the conditioned medium obtained by co-culture of stem cells and immune cells, it was determined that the melanin synthesis inhibitory effect was greater depending on the conditioned medium concentration. The amount of
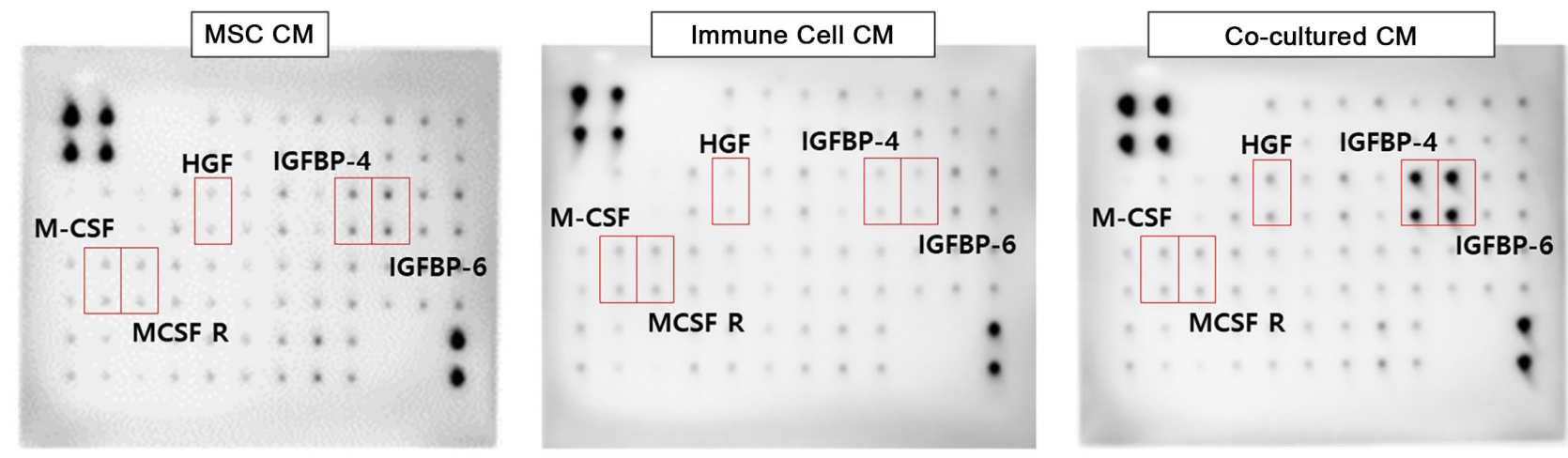

\begin{tabular}{cccccc}
\hline Growth Factors & MSC CM & Immune Cell CM & Co-cultured CM & MSC/mmune Cell ave. & Ratio \\
\hline HGF & 1077.3 & 818.8 & 1173.7 & 948.1 & $124 \%$ \\
IGFPB-4 & 3138.8 & 1786.0 & 5444.8 & 2462.4 & $221 \%$ \\
IGFPB-6 & 4248.0 & 2117.2 & 6682.8 & 3182.6 & $210 \%$ \\
M-SCF & 1476.1 & 2294.7 & 2707.9 & 1885.4 & $144 \%$ \\
MCSF R & 1397.4 & 2368.8 & 2531.3 & 1883.1 & $134 \%$ \\
\hline
\end{tabular}

Figure 2. Growth factor assay. In the case of the growth factor array, the protocol provided by the company was used. Briefly, each culture was reacted with a membrane, reacted with an antibody, and detected with chemidoc using HRP-streptavidin solution. Growth factors such as IGFPB were significantly increased in the conditioned medium obtained through co-culture of stem cells and immune cells than mono-culture.

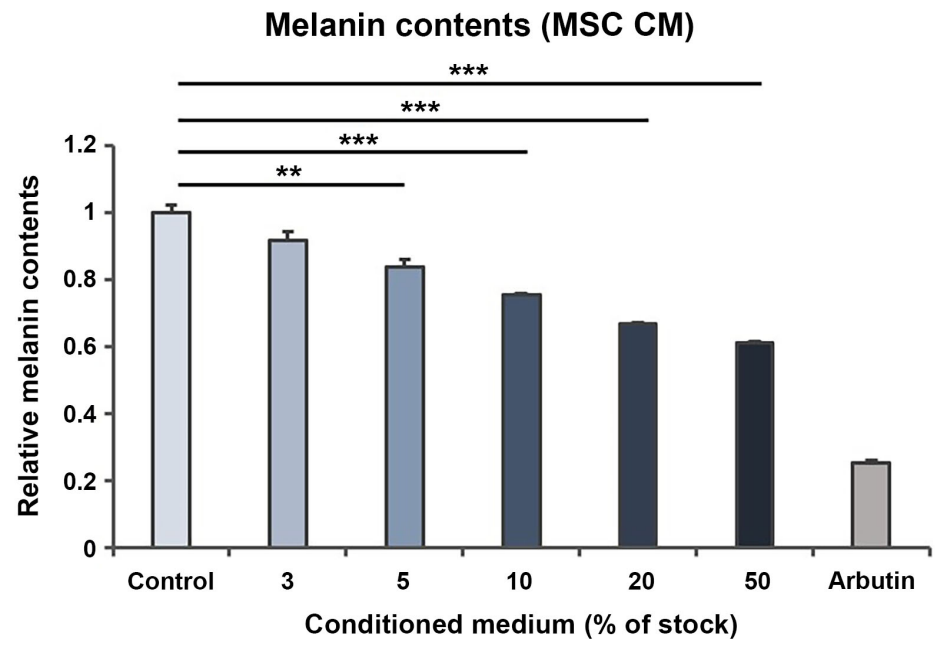

Figure 3. Melanin contents assay. B16F10 cells were cultured to an appropriate number, the culture medium was treated at the indicated concentration, and melanocytes were lysed with $1 \mathrm{~N} \mathrm{NaOH}$ at the endpoint to measure melanin contents at absorbance at 405 $\mathrm{nm}$. In the mono-cultured stem cells conditioned medium the melanin synthesis inhibitory effect was confirmed when it contained $3 \%$ of conditioned medium or more. 


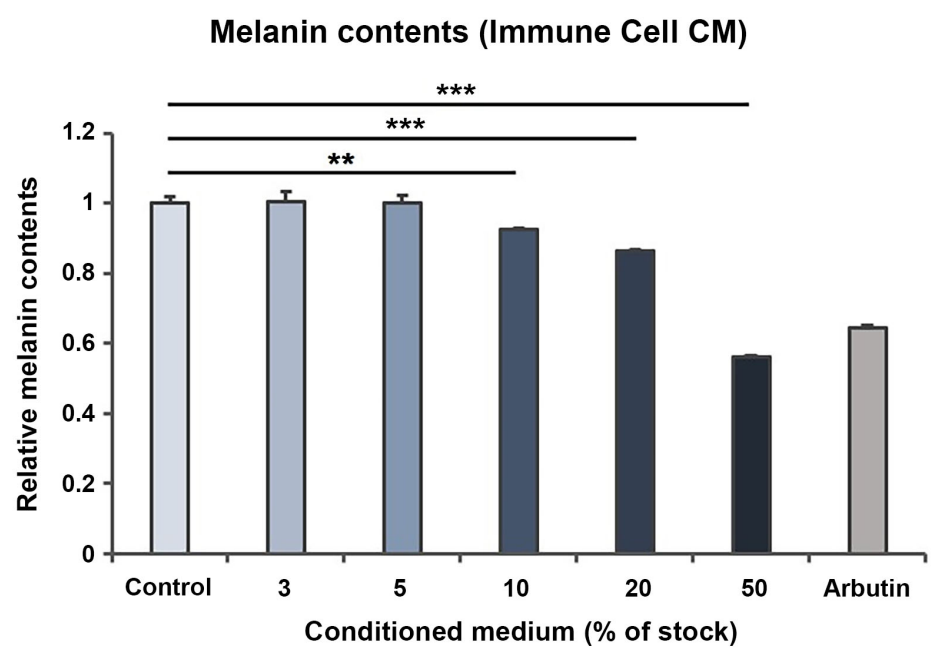

Figure 4. Melanin Contents assay. B16F10 cells were cultured to an appropriate number, the culture medium was treated at the indicated concentration, and melanocytes were lysed with $1 \mathrm{~N} \mathrm{NaOH}$ at the endpoint to measure melanin contents at absorbance at 405 $\mathrm{nm}$. In the mono-cultured immune cells conditioned medium, the melanin synthesis inhibitory effect was confirmed when it contained $20 \%$ of conditioned medium or more.

melanin syn-thesis significantly decreased to $59 \% \pm 2 \%$ even under the condition when the co-cultured conditioned medium contained only $3 \%$, and to $52 \%$ under the condition containing only $5 \%$. The amount of melanin synthesis decreased by $49 \% \pm 3 \%$ in the $10 \%$ co-cultured conditioned medium. In addition, it was verified that melanin synthesis was sharply down to $41 \% \pm 1 \%$ in the $20 \%$ co-cultured conditioned medium. It was verified that the amount of melanin synthesis decreased by $37 \% \pm 3 \%$ and $29 \% \pm 1 \%$ in the conditions of $50 \%$ co-cultured conditioned medium (Figure 5).

These decreased results of melanin synthesis were similar or superior to the reduction of $33 \% \pm 4 \%$ in arbutin which is one of the whitening agents.

To evaluate the whitening effects of three different conditioned medium, tyrosinase activity assay was performed. It was found that the tyrosinase activity reduced by $6 \pm 4 \%$ even only in $3 \%$ of stem cells conditioned medium.

Notably, at $5 \%$ the inhibitory activity of $48 \% \pm 2 \%$, equivalent to half, was confirmed, and a non-significant difference was seen with the $52 \% \pm 5 \%$ inhibitory activity value of the arbutin-treated group.

In addition, when it treated about $50 \%$ of conditioned medium, tyrosinase inhibitory activity was about $60 \% \pm 1 \%$, confirming an effect superior to $44 \% \pm 5 \%$ of the arbutin treated group (Figure 6).

In the case of immune cells conditioned medium, there was a slight change in tyrosinase activity at most concentrations. Through this result, it is thought that immune cells affect melanin synthesis through different pathways (Figure 7).

When the effect was determined with the co-culture, in the case of conditioned medium containing $5 \%$, the inhibitory activity was $17 \% \pm 9 \%$, at $20 \%$, a value of $44 \% \pm 5 \%$ equivalent to half was confirmed and there was no significant difference with the result of $48 \% \pm 5 \%$ of the arbutin treated group. 
When the conditioned medium contained $50 \%$, it exhibited an inhibitory activity of about $78 \% \pm 1 \%$, it showed an effect of $44.5 \%$, which was twice lower than that of the arbutin-treated group (Figure 8).

Through this, it was confirmed that the whitening effect had a greater effect when co-cultured than cultured with stem cells or immune cells alone.

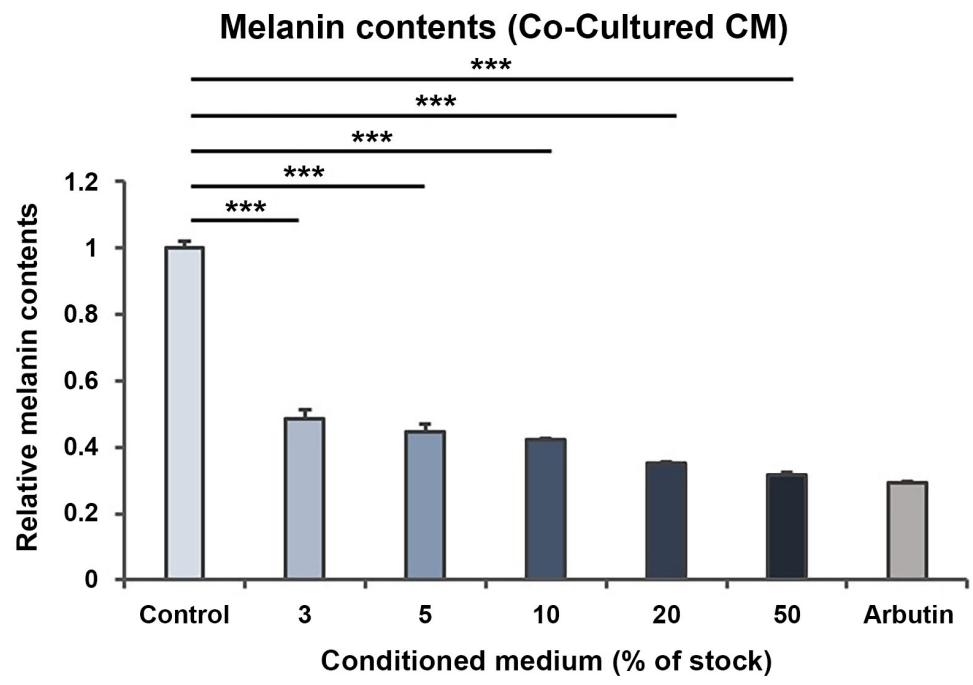

Figure 5. Melanin Contents assay. B16F10 cells were cultured to an appropriate number, the culture medium was treated at the indicated concentration, and melanocytes were lysed with $1 \mathrm{~N} \mathrm{NaOH}$ at the endpoint to measure melanin contents at absorbance at 405 $\mathrm{nm}$. In the co-culture conditioned medium, the melanin synthesis inhibitory effect increased more rapidly than in the mono-cultured conditioned medium treatment, showing a very large melanin contents reduction effect in the $3 \%$ treatment group.

Tyrosinase activity (MSC CM)

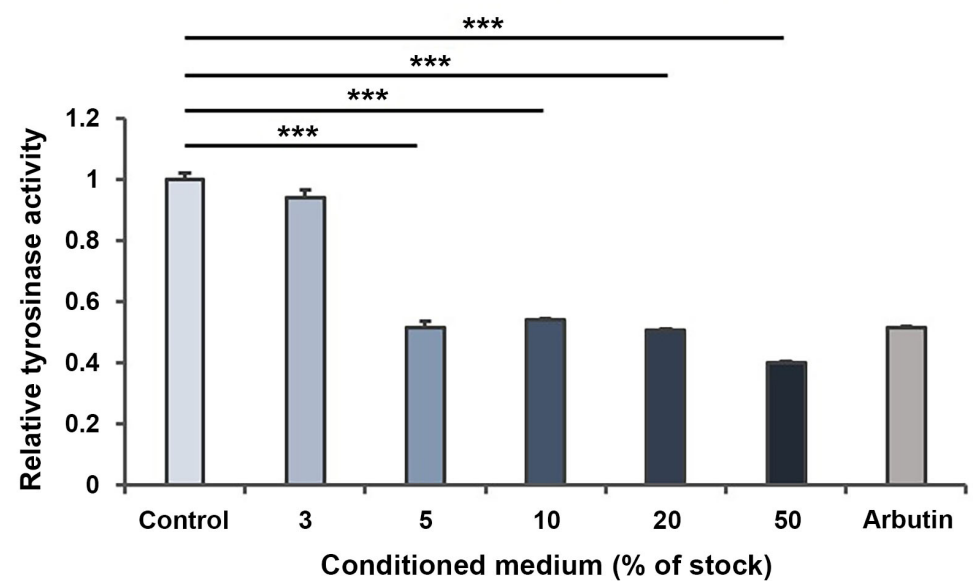

Figure 6. Tyrosinase inhibition activity assay. B16F10 cells were cultured to an appropriate number, the culture medium was treated at the indicated concentration, and melanocytes were lysed with $1 \mathrm{~N} \mathrm{NaOH}$ at the endpoint. The same amount of protein was fractionated from the cell lysate, incubated with L-DOPA, and the changed color was measured at absorbance at $405 \mathrm{~nm}$. The tyrosinase inhibitory activity rate of the stem cells conditioned medium was significantly decreased at $3 \%$, and its effect was similar to the positive control at $5 \%$. 
Tyrosinase activity (Immune Cell CM)

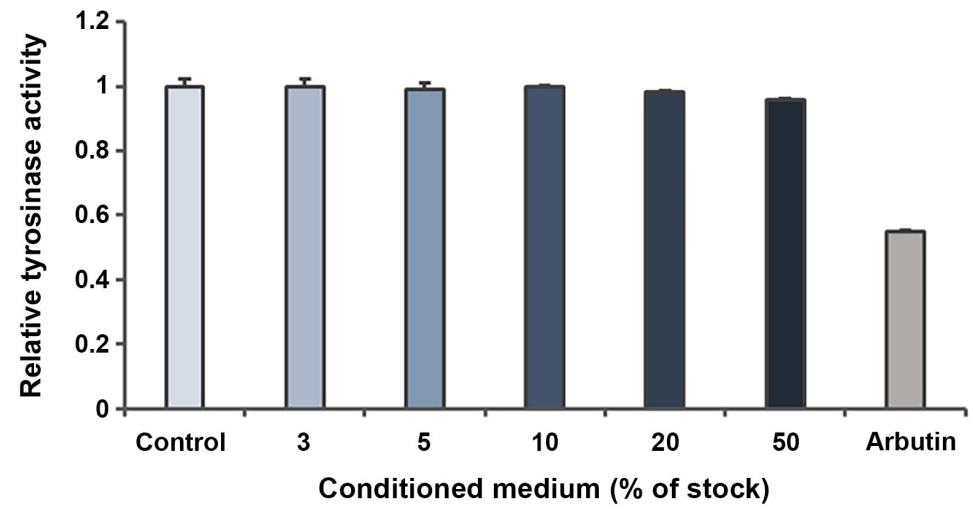

Figure 7. Tyrosinase inhibition activity assay. B16F10 cells were cultured to an appropriate number, the culture medium was treated at the indicated concentration, and melanocytes were lysed with $1 \mathrm{~N} \mathrm{NaOH}$ at the endpoint. The same amount of protein was fractionated from the cell lysate, incubated with L-DOPA, and the changed color was measured at absorbance at $405 \mathrm{~nm}$. The tyrosinase inhibitory activity rate of the Immune cells conditioned medium was barely changed at every concentration.

Tyrosinase activity (Co-cultured CM)

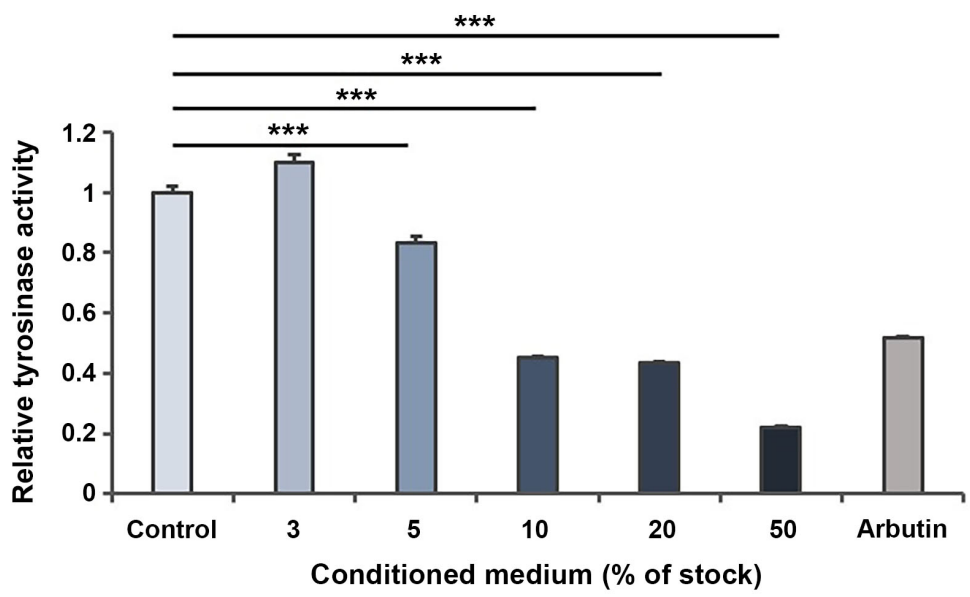

Figure 8. Tyrosinase inhibition activity assay. B16F10 cells were cultured to an appropriate number, the culture medium was treated at the indicated concentration, and melanocytes were lysed with $1 \mathrm{~N} \mathrm{NaOH}$ at the endpoint. The same amount of protein was fractionated from the cell lysate, incubated with L-DOPA, and the changed color was measured at absorbance at $405 \mathrm{~nm}$. The tyrosinase activity of the conditioned medium obtained through co-culture of stem cells and immune cells showed similar results to that of the positive control at $10 \%$, and the inhibitory effect was about twice that of the positive control at $50 \%$.

\section{Discussion}

Because of excellent whitening effects, a conditioned medium using stem cells or immune cells is widely used as a raw material for cosmetics. It is known that both stem cells and immune cells conditioned medium have an excellent effect on whitening [20]. There is a disadvantage that a large concentration of conditioned medium must be used to achieve a whitening effect although each condi- 
tioned medium is extensively used as a cosmetics material.

In this study, in order to solve such a limitation, both cells were co-cultured to add a synergistic effect to obtain a result that could produce an excellent whitening effect even when a small amount was added in cosmetics.

As a result of cytokine array of conditioned medium obtained through monoculture of stem cells or immune cells, it was confirmed that CCL1, CCL2, CCL5, CXCL8, etc. were additionally expressed in the conditioned medium obtained through co-culture, while those did not appear in mono-culture. These CCL1, CCL2, and CCL5 play the most important role in antimicrobial ingredients. IL-8 is one of the cytokines that participate in skin cells immunity. In addition, since they play various roles in anti-oxidative stress and angiogenesis, additional effects other than whitening can be expected [17].

According to the result of the growth factor array, it was verified that the expression patterns of cytokines are totally different. Expression of some factors including HGF and IGFPB-6 in the conditioned medium obtained through co-culture was found to increase significantly. HGF contributes to endothelial cells division, cells migration, and survival. It has an excellent whitening effect against UV-induced aging. Due to the multiple functions of HGF, it induces the migration and proliferation of melanin cells and finally separates them from the stratum corneum together with keratinocytes [18] [21] [22]. IGFPB-6 inhibits melanin formation through inhibiting oxidative action and aging of keratinocytes rather than acting directly on melanocytes [23].

Melanin synthesis is reduced in a concentration-dependent manner by various cytokines and growth factors contained in stem cells and immune cells conditioned medium. In a $3 \%$ conditioned medium of stem cells, melanin synthesis was reduced by $9 \% \pm 2 \%$. A significant decrease of melanin synthesis was observed depending on the concentration of the conditioned medium to $84 \% \pm 1 \%$ in $5 \%, 75 \% \pm 2 \%$ in $10 \%$, and $67 \% \pm 1 \%$ in $20 \%$ condition. When melanin contents were checked using immune cells conditioned medium, a decreasing tendency of the melanin synthesis rate was confirmed at $10 \%$, and a significant decrease at $20 \%$.

In the case of the conditioned medium obtained through the mono-cultured of each cell, it was confirmed that the melanin synthesis inhibition rate was reduced by $50 \%$ or more when it contained $50 \%$ or more. Markedly, when the co-cultured conditioned medium was treated 50\%, melanin contents showed $37 \% \pm 3 \%$, which is similar to $33 \% \pm 4 \%$ of arbutin, a whitening agent.

In the case of inhibition of tyrosinase activity, stem cells conditioned medium seemed to be more effective at the beginning, but it was corroborated that the effect of the co-cultured conditioned medium was higher from the time when the concentration of the co-cultured conditioned medium was $20 \%$.

The tyrosinase inhibitory activity rate of the immune cells conditioned medium did not show a significant result compared to the stem cells conditioned medium or the co-cultured conditioned medium. However, all conditioned media did not affect the viability of B16F10 (data not shown), so this clearly shows 
that co-cultured conditioned media has a synergistic effect.

To sum up, the experiment results indicate that whitening effects increase dependently as the concentration of the mono-cultured conditioned medium of stem cells or immune cells increases. Containing $10 \%$ of the co-cultured conditioned medium was found to be like the effect of positive control arbutin. If the conditioned medium contained $50 \%$ of the co-cultured conditioned medium, the whitening effect was much better than the positive control arbutin.

The development of cosmetics using cell culture has been in the spotlight recently, and regulations on human-derived cultures are being made in each country. In Korea, the Cosmetics raw regulates human-derived substances and manages them through various standards such as donor eligibility testing, cell or tissue collection and testing, and management of cultural facilities and environment. The culture medium produced in compliance with these regulations can be registered as a cosmetic ingredient by registering in INCI, etc. Currently, the use of cosmetics for human materials is not too difficult to develop as long as the regulations are well followed, and the co-cultured medium obtained through this study is also the same.

The co-culture system suggests that even a small amount of co-culture conditioned medium can have a great effect in the cosmetic market for whitening using conditioned medium. And by using the synergistic effect of stem cells and immune cells to secrete substances with more efficacy, it is thought that various applications, as well as cosmetic raw materials, are possible. In the case of co-cultured CM conducted in this paper, only the whitening effect was shown, but additional research is needed on how to have the whitening effect. In addition to whitening, it is judged that it is necessary to study the effects of wrinkle improvement and skin barrier improvement.

\section{Acknowledgements}

The authors are thankful to Immunisbio. Co., Ltd. for providing the experiments of Immune cells.

\section{Conflicts of Interest}

The authors declare no conflicts of interest regarding the publication of this paper.

\section{References}

[1] Samara Pollock, B.A. (2021) The Dark Side of Skin Lightening: An International Collaboration and Review of a Public Health Issue Affecting Dermatology. International Journal of Women's Dermatology, 7, 158-164. https://doi.org/10.1016/j.ijwd.2020.09.006

[2] Costin, G.-E. (2007) Human Skin Pigmentation: Melanocytes Modulate Skin Color in Response to Stress. The FASEB Journal, 21, 976-994. https://doi.org/10.1096/fj.06-6649rev

[3] Moreiras, H. (2021) Melanin Transfer in the Epidermis: The Pursuit of Skin Pig- 
mentation Control Mechanisms. International Journal of Molecular Sciences, 22, Article No. 4466. https://doi.org/10.3390/ijms22094466

[4] Kim, H.J. (2020) A Study on Clinical Effectiveness of Cosmetics Containing Human Stem Cell Conditioned Media. Biomedical Dermatology, 4, Article No. 9. https://doi.org/10.1186/s41702-020-0056-9

[5] Hsu, J.-Y., et al. (2020) Anti-Melanogenesis Effects of Lotus Seedpod in Vitro and in Vivo. Nutrients, 12, 3535. https://doi.org/10.3390/nu12113535

[6] Chakraborty, A.K. (1998) Effect of Arbutin on Melanogenic Proteins in Human Melanocytes. Pigment Cell Research, 11, 206-212.

https://doi.org/10.1111/j.1600-0749.1998.tb00731.x

[7] Chen, L.W., et al. (2008) Paracrine Factors of Mesenchymal Stem Cells Recruit Macrophages and Endothelial Lineage Cells and Enhance Wound Healing. PLOS ONE, 13, e1886. https://doi.org/10.1371/journal.pone.0001886

[8] Park, B.S., et al. (2010) Hair Growth Stimulated by Conditioned Medium of Adipose Derived Stem Cells Is Enhanced by Hypoxia. Biomedical Research, 31, 27-34. https://doi.org/10.2220/biomedres.31.27

[9] Kim, W.-S., et al. (2007) Wound Healing Effect of Adipose-Derived Stem Cells: A Critical Role of Secretory Factors on Human Dermal Fibroblasts. Journal of Dermatological Science, 48, 15-24. https://doi.org/10.1016/j.jdermsci.2007.05.018

[10] Li, J.L., et al. (2012) Anti-Oxidant and Anti-Skin-Aging Effects of Abalone Viscera Extracts in Human Dermal Fibroblasts. Korean Journal of Food Preservation, 19, 463-469. https://doi.org/10.11002/kjfp.2012.19.4.463

[11] Kim, W.-S., et al. (2009) Antiwrinkle Effect of Adipose-Derived Stem Cell: Activation of Dermal Fibroblast by Secretory Factors. Journal of Dermatological Science, 53, 96-102. https://doi.org/10.1016/j.jdermsci.2008.08.007

[12] Kim, W.-S., et al. (2008) Whitening Effect of Adipose-Derived Stem Cells: A Critical Role of TGF-b 1. Biological and Pharmaceutical Bulletin, 31, 606-610. https://doi.org/10.1248/bpb.31.606

[13] Zuk, P.A., et al. (2001) Multilineage Cells from Human Adipose Tissue: Implications for Cell-Based Therapies. Tissue Engineering, 7, 211-228. https://doi.org/10.1089/107632701300062859

[14] Hwang, I. (2017) Neural Stem Cells and Its Derivatives as a New Material for Melanin Inhibition. International Journal of Molecular Sciences, 19, Article No. 36. https://doi.org/10.3390/ijms19010036

[15] Kim, D.S., et al. (2021) Potential of Natural Killer Cell Enriched Conditioned Media for Skin Care and Anti-Aging. Journal of Cosmetics, Dermatological Sciences and Applications, 11, 123-139. https://doi.org/10.4236/jcdsa.2021.112013

[16] Lee, Y.-H., et al. (2005) Chemical Stability and Whitening Activity of Mixture of Functional Ingredients. Journal of the Society of Cosmetic Scientists of Korea, 31, 295-304.

[17] Saghaie, L., et al. (2013) Synthesis and Tyrosinase Inhibitory Properties of Some Novel Derivatives of Kojic Acid. Research in Pharmaceutical Sciences, 8, 233-242.

[18] Costin, G.E. (2007) Human Skin Pigmentation: Melanocytes Modulate Skin Color in Response to Stress. FASEB Journal, 21, 976-994. https://doi.org/10.1096/fj.06-6649rev

[19] Njar, M. (2018) Mesenchymal Stromal Cells of the Bone Marrow and Natural Killer Cells: Cell Interactions and Cross Modulation. Journal of Cell Communication and Signaling, 12, 673-688. https://doi.org/10.1007/s12079-018-0448-4 
[20] Amirthlingam, M. (2016) Stem Cell Derived Cosmetic Products: An Overview. Manipal Journal of Medical Sciences, 1, 46-52.

[21] Halaban, R. (1993) Pigmentation and Proliferation of Human Melanocytes and the Effects of Melanocyte-Stimulating Hormone and Ultraviolet Blight. Annals of the New York Academy of Sciences, 680, 290-301.

https://doi.org/10.1111/j.1749-6632.1993.tb19691.x

[22] Matsumoto, K. (1991) Hepatocyte Growth Factor Is a Potent Stimulator of Human Melanocyte DNA Synthesis and Growth. Biochemical and Biophysical Research Communications, 176, 45-51. https://doi.org/10.1016/0006-291X(91)90887-D

[23] Conese, M. (2020) Insulin-Like Growth Factor Binding Protein 6 Is Secreted in Extracellular Vesicles upon Hyperthermia and Oxidative Stress in Dendritic Cells But Not in Monocyte. International Journal of Molecular Sciences, 21, Article No. 4428. https://doi.org/10.3390/ijms21124428 